\title{
THE IRRATIONALITY OF CERTAIN INFINITE SERIES
}

\author{
by C. BADEA
}

(Received 14 February, 1986)

The aim of this paper is to prove the irrationality of a certain class of infinite series. The main theorem is related to some results due to Erdös and Straus [7], Erdös [5] and Sándor [15]. As applications of the main result, the solutions of two problems posed by Erdös and Graham [6] are given, among others.

1. Introduction. Some criteria for the irrationality of infinite series of positive rationals have been proved. In the case when an infinite series is divergent, the problem of the irrationality of its value does not arise. Thus we shall assume in what follows that all infinite series which appear are convergent. Another way in which we can avoid this trivial case is to make the convention that $\infty$ is an irrational number (or, perhaps more naturally, to consider that $\infty$ is irrational and rational at the same time).

The purpose of this paper is to prove the irrationality of a certain class of infinite series, i.e. to give another criterion for irrationality. Our main result is related to the following known theorems.

THEOREM A (Erdös and Straus [7]). Let $\left(a_{n}\right), n \geqslant 1$, be a sequence of positive integers such that

$$
a_{n+1} \geqslant a_{1} a_{2} \ldots a_{n}
$$

for each $n$ and, for every $C>0$, there is a natural number $n>C$ with the property that

$$
a_{n+1} \neq a_{n}^{2}-a_{n}+1 \text {. }
$$

Then the sum of the series $\sum_{n=1}^{\infty} 1 / a_{n}$ is an irrational number.

THEOREM B (Erdös [5]). Let $a_{1}<a_{2}<\ldots<a_{n}<\ldots$ be a sequence of positive integers such that

$$
\limsup _{n \rightarrow \infty} a_{n}^{1 / 2^{n}}=\infty
$$

and

$$
a_{n}>n^{1+\varepsilon}
$$

for a number $\varepsilon>0$ and for every $n>n_{0}(\varepsilon)$. Then the sum of the series $\sum_{n=1}^{\infty} 1 / a_{n}$ is an irrational number.

THEOREM C (Sándor [15]). Let $\left(a_{n}\right),\left(b_{n}\right), n \geqslant 1$, be two sequences of positive integers Glasgow Math. J. 29 (1987) 221-228. 
such that

and

$$
\limsup _{n \rightarrow \infty} \frac{a_{n+1}}{a_{1} a_{2} \ldots a_{n}} \cdot \frac{1}{b_{n+1}}=\infty
$$

$$
\liminf _{n \rightarrow \infty} \frac{a_{n+1}}{a_{n}} \cdot \frac{b_{n}}{b_{n+1}}>1 \text {. }
$$

Then the sum of the series $\sum_{n=1}^{\infty} b_{n} / a_{n}$ is an irrational number.

In the proof of the main result we shall use a criterion for irrationality of limits of rationals due to Brun [3]. As applications we give the solutions of two problems recently posed by Erdös and Graham [6] and we prove that every convergent infinite series of positive rationals has infinitely many disjoint subseries (to be defined) with irrational sums.

We note in ending that the same method of proof for the main result was used [1] to obtain some criteria for the irrationality of certain infinite products.

2. Main result. The main result of the present paper is the following.

THEOREM. Let $\left(b_{n}\right)$ and $\left(a_{n}\right), n \geqslant 1$, be two sequences of positive integers such that the relation

$$
a_{n+1}>\frac{b_{n+1}}{b_{n}} a_{n}^{2}-\frac{b_{n+1}}{b_{n}} a_{n}+1
$$

holds for every large $n$. Then the sum of the series $\sum_{n=1}^{\infty} b_{n} / a_{n}$ is an irrational number.

Proof. We have

$$
\sum_{n=1}^{\infty} \frac{b_{n}}{a_{n}}=\lim _{n \rightarrow \infty}\left(\frac{b_{1}}{a_{1}}+\ldots+\frac{b_{n}}{a_{n}}\right)=\lim _{n \rightarrow \infty} \frac{A_{n}}{P_{n}}
$$

where, for every $n \geqslant 1, P_{n}=a_{1} a_{2} \ldots a_{n}$ and $A_{n}=\sum_{j=1}^{n} b_{j} P_{n} / a_{j}$.

Brun's criterion asserts that a positive real number $\alpha$ which is the limit of an increasing sequence of rationals

$$
\alpha=\lim _{n \rightarrow \infty} \frac{y_{n}}{x_{n}}
$$

where $x_{n}$ and $y_{n}, n \geqslant 1$, are positive integers, is irrational if

$$
\frac{y_{n+2}-y_{n+1}}{x_{n+2}-x_{n+1}}<\frac{y_{n+1}-y_{n}}{x_{n+1}-x_{n}}
$$

for all large $n$. 
Taking into account this theorem, we shall prove the inequality (4) for $y_{n}=A_{n}$ and $x_{n}=P_{n}$. Because $b_{n}$ and $a_{n}$ are positive integers for all $n$, we get that the sequence $\left(A_{n} / P_{n}\right), n \geqslant 1$, is increasing and thus, via Brun's theorem, we shall find that the sum of the series $\sum_{n=1}^{\infty} b_{n} / a_{n}$ is irrational.

Firstly, we derive a recurrence relation for the sequence $\left(A_{n}\right)$. Because $P_{n+1}=$ $a_{n+1} P_{n}$, we have

$$
A_{n+1}-A_{n}=a_{n+1} \sum_{i=1}^{n+1} P_{n}\left(b_{i} / a_{i}\right)-\sum_{i=1}^{n} P_{n}\left(b_{i} / a_{i}\right)
$$

so we may write the following relation

$$
A_{n+1}=a_{n+1} A_{n}+b_{n+1} P_{n} .
$$

Using (5) in the inequality (4) with $y_{n}=A_{n}$ and $x_{n}=P_{n}$, we obtain the following equivalent inequality

$$
\frac{\left(a_{n+2}-1\right) A_{n+1}+b_{n+2} P_{n+1}}{P_{n+1}\left(a_{n+2}-1\right)}<\frac{\left(a_{n+1}-1\right) A_{n}+b_{n+1} P_{n}}{P_{n}\left(a_{n+1}-1\right)}
$$

which is also equivalent to

$$
\frac{A_{n+1}}{P_{n+1}}-\frac{A_{n}}{P_{n}}<\frac{b_{n+1}}{a_{n+1}-1}-\frac{b_{n+2}}{a_{n+2}-1} .
$$

Using again (5), we have

$$
\frac{A_{n+1}}{P_{n+1}}-\frac{A_{n}}{P_{n}}=\frac{A_{n+1} P_{n}-A_{n} P_{n+1}}{P_{n} P_{n+1}}=\frac{A_{n+1}-a_{n+1} A_{n}}{P_{n+1}}=\frac{b_{n+1}}{a_{n+1}} .
$$

Therefore the inequality (6) is equivalent to

$$
\frac{b_{n+1}}{a_{n+1}}<\frac{b_{n+1} a_{n+2}-b_{n+1}-a_{n+1} b_{n+2}+b_{n+2}}{\left(a_{n+1}-1\right)\left(a_{n+2}-1\right)} .
$$

From (7), we deduce by routine calculations the following relation

$$
b_{n} a_{n+1}>b_{n+1} a_{n}^{2}-b_{n+1} a_{n}+b_{n},
$$

which is just (1).

Thus it follows that (4) holds for every sufficiently large $n$ and, via Brun's criterion, the proof is now complete.

Remark. A generalization of Brun's irrationality criterion was given by Froda [8]. Namely, Froda proved that Brun's criterion is also true if $y_{n}$ and $x_{n}$ are positive real numbers so that (4) holds. The same method of the proof of the above theorem remains valid to show, with the help of Froda's generalization, that the main result is also true for positive numbers $a_{n}$ and $b_{n}$. However, this is not valid because Froda's generalization is 
not correct. Indeed, we gave recently [1] the following counter-example: let us define the sequence $\left(c_{n}\right)$ by $c_{1}=2$ and the recursive relation

$$
c_{n+1}=c_{n}^{2}-c_{n}+1
$$

then the sequences

$$
y_{n}=\prod_{i=1}^{n}\left(a_{i}+\log 1.5\right) \quad \text { and } \quad x_{n}=\prod_{i=1}^{n} a_{i} \text {, }
$$

where $\left(a_{n}\right)$ is given by $a_{n}=(\log 1.5) /\left(2^{1 / c_{n}}-1\right)$, provide the desired counter-example.

3. Some consequences. In this section we shall give some easy consequences of the main result which show the similarity (and the differences) of our theorem with the above-mentioned results of Erdös and Straus, Erdös and Sándor.

The following result is an easy consequence of the main theorem and is related to Theorem A of Erdös and Straus.

COROLlaRY 1. Let $\left(a_{n}\right), n \geqslant 1$, be a sequence of positive integers such that

$$
a_{n+1}>a_{n}^{2}-a_{n}+1
$$

holds for all large $n$. Then the sum of the series $\sum_{n=1}^{\infty} 1 / a_{n}$ is an irrational number.

Proof. Put $b_{n}=1$ in the above theorem.

Remark. In a certain sense, Corollary 1 is the best possible. Indeed, for the sequence $\left(c_{n}\right)$ defined in the remark in the previous section, we have $\sum_{n=1}^{k} 1 / c_{n}=$ $1-\left(c_{k+1}-1\right)^{-1}$ and thus $\sum_{n=1}^{\infty} 1 / c_{n}=1$; so we cannot replace (8) by $a_{n+1} \geqslant a_{n}^{2}-a_{n}+1$. This example also shows that the answer to the last question of Problem E.24 in [11] is negative.

We recall that the sequence $\left(u_{n}\right), n \geqslant 1$, is eventually nondecreasing if $u_{n+1} \geqslant u_{n}$ for all large $n$.

In connection with Theorem B of Erdös we now state another corollary.

Corollary 2. Let $\left(a_{n}\right), n \geqslant 1$, be a sequence of positive integers such that the sequence $\left(a_{n}^{1 / 2^{n}}\right), n \geqslant 1$, is eventually nondecreasing. Then the sum of the series $\sum_{n=1}^{\infty} 1 / a_{n}$ is an irrational number.

Proof. Because the sequence $\left(a_{n}^{1 / 2^{n}}\right), n \geqslant 1$, is eventually nondecreasing, we have

$$
a_{n+1} \geqslant a_{n}^{2}
$$


for all large $n$. Using the convention that $\sum_{n=1}^{\infty} 1 / a_{n}$ is a convergent series (see the first section), we get that $a_{n}$ tends to infinity with $n$ and thus

for all large $n$.

$$
a_{n}>1
$$

We complete the proof by using (9), (10) and Corollary 1.

Considering again series of the form $\sum_{n=1}^{\infty} b_{n} / a_{n}$, we may state the following result which is related to Sándor's Theorem C.

COROLLARY 3. Let $\left(b_{n}\right)$ and $\left(a_{n}\right), n \geqslant 1$, be two sequences of positive integers such that the sequence $\left(a_{n+1} / b_{n+1} a_{1} a_{2} \ldots a_{n}\right), n \geqslant 1$, is eventually nondecreasing. Then the sum of the series $\sum_{n=1}^{\infty} b_{n} / a_{n}$ is an irrational number.

Proof. The condition from the statement of this corollary is equivalent to

$$
a_{n+1} \geqslant \frac{b_{n+1}}{b_{n}} a_{n}^{2}
$$

Now we can complete the proof as in the proof of Corollary 2 using (11), the convention from the first section and the main result.

As a general remark, we note that in all these consequences something is gained and something is lost from the results mentioned in Theorems A to C.

4. Disjoint subseries with irrational sums. We say that $\sum_{n=1}^{\infty} v_{n}$ is a subseries of a given series $\sum_{n=1}^{\infty} u_{n}$ if $\left(v_{n}\right)$ is a subsequence of the sequence $\left(u_{n}\right)$. Two subseries $\sum_{n=1}^{\infty} v_{n}$ and $\sum_{n=1}^{\infty} w_{n}$ of the same series $\sum_{n=1}^{\infty} u_{n}$ are disjoint if $\left(w_{n}\right)$ is a subsequence of the sequence $\left(u_{n}\right)$ from which has been taken the subsequence $\left(v_{n}\right)$, i.e. $\left(w_{n}\right)$ and $\left(v_{n}\right)$ are disjoint subsequences of the sequence $\left(u_{n}\right)$.

As an application of the main result we shall prove the following (perhaps unexpected) proposition.

Proposition. Every convergent infinite series of positive rationals has infinitely many disjoint subseries with irrational sums.

This result is similar to a proposition in [1] , where we proved that every convergent infinite product of rational numbers greater than 1 has infinitely many disjoint subproducts with irrational values (the notions of subproducts and disjoint subproducts are defined similarly). 
Proof of proposition. Let $\sum_{n=1}^{\infty} b_{n} / a_{n}$ be a convergent series of positive rationals. Then the sequence $\left(b_{n} / a_{n}\right)$ tends to zero when $n$ tends to infinity. Hence there are infinitely many disjoint subsequences $\left(a_{n(k)}^{(p)}\right)$ and $\left(b_{n(k)}^{(p)}\right), k \geqslant 1$, of the sequences $\left(a_{n}\right)$ and $\left(b_{n}\right)$ respectively, $p=1,2, \ldots$, such that

$$
a_{n(k+1)}^{(p)}>\frac{b_{n(k+1)}^{(p)}}{b_{n(k)}^{(p)}}\left(a_{n(k)}^{(p)}\right)^{2}
$$

for all large $k$ because the sequence $\left(a_{n}\right)$ tends to infinity with $n$.

Now, using Corollary 3 , we find that the subseries of the series $\sum_{n=1}^{\infty} b_{n} / a_{n}$ generated by the subsequences $\left(a_{n(k)}^{(p)}\right)$ and $\left(b_{n(k)}^{(p)}\right)$ have irrational sums.

The proof is complete.

REMARK. There is (see [4]) a convergent series of positive rationals such that all its subseries have irrational sums. This shows that we cannot replace the word irrational by rational in the above proposition. This contrasts with the known fact [9] that if $\sum_{n=1}^{\infty} a_{n}$ is a divergent series with $a_{n} \rightarrow 0$ as $n \rightarrow \infty$ then every positive real number may be written as the sum of a convergent subseries of $\sum_{n=1}^{\infty} a_{n}$. The above "negative" result for series may be explained by the fact that the set of rational numbers is denumerable while the set of irrationals is uncountable. We note that in [1] we posed the question of the existence of a convergent infinite product of rational numbers greater than 1 such that all its subproducts have irrational values and, as far as we know, the problem is still open.

5. Two problems of Erdös and Graham. In this section we shall give, based upon our irrationality criterion, the solutions of two problems posed by Erdös and Graham [6, pp. 64-65].

It has been noted that for the sequence of Fibonacci numbers $F_{n}$ defined by $F_{0}=0$, $F_{1}=1, F_{n+2}=F_{n+1}+F_{n}, n \geqslant 0$, we have (see [10], [12])

$$
\sum_{n=0}^{\infty} \frac{1}{F_{2^{n}}}=\frac{7-\sqrt{ } 5}{2}
$$

and thus the sum of the above series is irrational. This is not surprising in view of the above proposition. As a related result, we mention the transcendence of the sum of the series

$$
\sum_{n=0}^{\infty} \frac{1}{n ! F_{2^{n}}}
$$

which was independently proved by Mignotte [14] and Mahler [13].

In their very nice book [6, pp. 64-65], Erdös and Graham wrote: "However, nothing 
is known about the character of the related sums

$$
\sum_{n=1}^{\infty} \frac{1}{F_{2^{n}+1}} \text { or } \sum_{n=1}^{\infty} \frac{1}{L_{2^{n}}} .
$$

Here $L_{n}$ is the $n$th Lucas number, defined by $L_{n}=F_{n-1}+F_{n+1}$."

The aim of this section is to prove the above series have irrational sums. Firstly we prove the following corollary.

COROLlary 4. The sum of the series $\sum_{n=1}^{\infty} 1 / F_{2^{n}+1}$ is an irrational number.

In the proof of this (and the next) result we shall use some known identities for Fibonacci numbers which can be easily proved by induction.

Proof of Corollary 4. Keeping in mind the first corollary, it is sufficient to prove that

$$
F_{2^{n+1}+1}>F_{2^{n}+1}^{2}-F_{2^{n}+1}+1
$$

or even

$$
F_{2^{n+1}+1} \geqslant F_{2^{n}+1}^{2}
$$

for all large $n$. But this follows from $F_{2 k+1}=F_{k}^{2}+F_{k+1}^{2}$ for $k=2^{n}$ and now the proof is complete.

For the second problem of Erdös and Graham we prove the following corollary.

COROLlary 5. The sum of the series $\sum_{n=1}^{\infty} 1 / L_{2^{n}}$ is an irrational number.

Proof. Firstly we prove that, for all large $p$, we have

$$
L_{2 p}>L_{p}^{2}-L_{p}+1
$$

The above inequality (12) is equivalent to

$$
F_{2 p+1}+F_{2 p-1}>F_{p+1}^{2}+F_{p-1}^{2}+2 F_{p+1} F_{p-1}-F_{p+1}-F_{p-1}+1
$$

or, by the identity $F_{2 k+1}=F_{k}^{2}+F_{k+1}^{2}$, to

$$
F_{p+1}+F_{p-1}+2\left(F_{p}^{2}-F_{p+1} F_{p-1}\right)>1 \text {. }
$$

But $F_{p}^{2}-F_{p+1} F_{p-1}=(-1)^{p+1}$; so (13) holds for all large $p$. By putting $p=2^{n}$ in (12), we get

$$
L_{2^{n+1}}>L_{2^{n}}^{2}-L_{2^{n}}+1
$$

for all large $n$ and from Corollary 1 we deduce the desired conclusion.

REMARK. Recently [2] further contributions on these and other related problems of Erdös and Graham were given.

I am very grateful to the referee for helpful suggestions. 


\section{REFERENCES}

1. C. Badea, The irrationality of certain infinite products, to appear in Studia Univ. Babes-Bolyai Math.

2. C. Badea, On some problems of Erdös and Graham concerning Fibonacci and Lucas numbers, submitted.

3. V. Brun, Ein Satz über Irrationalität, Arch. for Math. og Naturvidenskab (Kristiania) 31 (1910), 3.

4. D. Călin and V. Kiss, Irationalitatea sumelor unor serii remarcabile, Gaz. Mat. Ser. A 75 (1970), 161-164.

5. P. Erdös, Some problems and results on the irrationality of the sum of infinite series, $J$. Math. Sci. 10 (1975), 1-7.

6. P. Erdös and R. L. Graham, Old and new problems and results in combinatorial number theory (Imprimerie Kunding, 1980).

7. P. Erdös and E. G. Straus, On the irrationality of certain Ahmes series, J. Indian Math. Soc. 27 (1963), 129-133.

8. A. Froda, Sur l'irrationalité du nombre $2^{e}$, Atti. Accad. Naz. Lincei Rend. Cl. Sci. Fis. Mat. Natur. (8) 35 (1963), 472-478.

9. B. Gelbaum, Problems in analysis (Springer, 1982).

10. I. J. Good, A reciprocal series of Fibonacci numbers, Fibonacci Quart. 12 (1974), 346.

11. R. K. Guy, Unsolved problems in number theory (Springer, 1981).

12. V. E. Hoggatt, Jr. and M. Bicknell, A reciprocal series of Fibonacci numbers with subscripts of $2^{n} k$, Fibonacci Quart. 14 (1976), 453-455.

13. K. Mahler, On the transcendency of the solutions of a special class of functional equations, Bull. Austral. Math. Soc. 13 (1975), 389-410.

14. M. Mignotte, Quelques problèmes d'effectivité en théorie des nombres (Thesis, Univ. Paris XIII, Paris, 1974). 3-12.

15. J. Sándor, Some classes of irrational numbers, Studia Univ. Babes-Bolyai Math. 29 (1984),

Department of Mathematics

UNIVERSITY OF CRAIOVA

1100 Craiova

ROMANIA 